\title{
Performance of the HEPD-02 LYSO calorimeter and expected sensitivity to GRBs detection
}

\section{Stefania Perciballi, ${ }^{a, *}$ Roberto luppa,,${ }^{b, c}$ Marco Mese,${ }^{e}$ Francesco Nozzoli, ${ }^{b}$ Giuseppe Osteria, ${ }^{d}$ Valentina Scotti ${ }^{d, e}$ and Pietro Ubertini ${ }^{f}$}

${ }^{a}$ Physics Department, Torino University,

Via Pietro Giuria, 1, Torino, Italy

${ }^{b}$ Istituto Nazionale Fisica Nucleare (INFN) - TIFPA,

Via Sommarive, 14, Trento, Italy

${ }^{c}$ Physics Department, Trento University,

Via Sommarive, 14, Trento, Italy

${ }^{d}$ Istituto Nazionale Fisica Nucleare (INFN),

Complesso universitario di Monte S. Angelo ed. 6, via Cinthia, Napoli, Italy

e Physics Department, Napoli University,

Via Cinthia, 21 , Napoli, Italy

${ }^{f}$ Istituto di Astrofisica e Planetologia Spaziali, INAF,

Via del fosso del cavaliere 100, Roma, Italy

E-mail: stefania.perciballi@edu.unito.it

A High-Energy Particle Detector will be launched on board the CSES-02 satellite with the main purpose of deepening our comprehension of the Earth magnetosphere, investigating the Inner Van Allen belts and contributing to the understanding of sub-GeV cosmic rays.

HEPD-02 is composed of two segmented scintillator layers for the trigger, a silicon pixel tracker and a calorimeter. The detector is surrounded by a plastic scintillator veto, except for the front side.

The calorimeter is composed of twelve layers of $1.1 \times 15 \times 15 \mathrm{~cm}^{3}$ plastic scintillator pads and two layers of 3 LYSO bars, as large as $2.5 \times 4.9 \times 15 \mathrm{~cm}^{3}$, disposed orthogonally to each other. Thanks to its features, HEPD-02 could identify gamma-rays converting in the LYSO crystals by requiring all the other sub-detectors to act as a veto.

In particular, Gamma Ray Bursts (GRBs) are extremely energetic events generated billions of light-years away from the Earth, possibly during neutron star mergers or supernova explosions. HEPD-02 would join the fleet of space detectors continuously monitoring the sky and searching for GRBs, widening the sky coverage and contributing to the knowledge of these phenomena. We report the measured radioactive background of a HEPD LYSO crystal due to the decay of ${ }^{176} \mathrm{Lu}\left(2.6 \%\right.$ ab., $\left.T_{1 / 2}=3.78 \times 10^{10} y\right)$, then describing a possible triggering scheme for GRBs and the expected sensitivity to GRBs in the $2-20 \mathrm{MeV}$ energy window.

$3^{\text {th }}$ International Cosmic Ray Conference (ICRC 2021)

July 12th - 23rd, 2021

Online - Berlin, Germany

\footnotetext{
*Presenter
} 


\section{The HEPD-02 detector}

HEPD-02 is a particle detector, designed and produced by the Limadou Collaboration, that will fly, among other payloads, on board the China Seismo - Electromagnetic Satellite (CSES-02) with the aim of better understanding the near-Earth electromagnetic environment, studying some phenomena in solar-terrestrial interaction and improving our knowledge of cosmic rays.

HEPD-02 is composed by two segmented trigger planes, a silicon pixel tracker and a calorimeter; the detector is covered by a veto system, except for the front side. The first trigger plane is made of a plastic scintillator divided in 5 different counters, while the second has 4 counters. The tracker is composed by 3 layers of silicon pixel detector and is placed between the two trigger planes. The calorimeter is made of 12 plastic scintillator layers with, at the bottom, 2 layers each consisting of 3 LYSO bars $\left(2.5 \times 4.9 \times 15 \mathrm{~cm}^{3}\right)$ deployed orthogonal to each other.

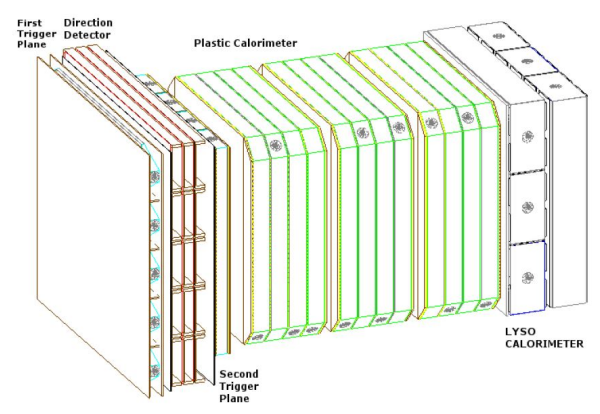

Figure 1: Schematic view of HEPD-02 sensitive units, the gray dots correspond to photo-multiplier tubes (PMTs) used for collecting the scintillation light. [1])

The scientific community is interested in the possibility of using the LYSO calorimeter to detect Gamma Ray Bursts (GRBs), in order to extend the sky coverage. The expected signature of GRBs events in the HEPD-02 detector requires a signal in the LYSO bars and the anticoincidence with all the other detecting units.

\section{LYSO for GRB detection}

Gamma Ray Bursts (GRBs) are one of the most energetic events in our Universe. They are originated during nearby supernovae explosions (long GRBs, lasting more than $2 \mathrm{~s}$ ) or during neutron star mergers up to redshift $z=8$ (short GRBs, lasting less than $2 \mathrm{~s}$ )[2]. Supernova explosions occur when a supernova starts burning iron and pressure from nuclear processes becomes insufficient to contrast the gravitational force. This leads to the star collapse and the subsequent explosion with the emission of GRBs from the poles. Quite oppositely, in the final phase of neutron stars merging, neutron capture takes place inside pieces of ejected material and it often causes a radioactively powered GRB.

Many space missions, i.e. Swift (NASA), Integral (ESA), Fermi (NASA) are currently monitoring the sky, observing GRBs to better understand these phenomena. The standard crystal for GRBs detection is BGO [3], $\mathrm{Bi}_{4} \mathrm{Ge}_{3} \mathrm{O}_{12}$, an inorganic scintillator with high density $\left(7.13 \mathrm{~g} / \mathrm{cm}^{3}\right)$, slow decay time (300 ns) and low intrinsic radioactivity $(1-10 \mathrm{~Bq} / \mathrm{kg})[4]$. 
We observe that ongoing space missions do not provide $100 \%$ duty-cycle full sky-coverage yet, so that increasing the wealth of space-based instrumentation sensitive to GRBs may improve the quantity and the quality of observations. In this respect, some interest has been expressed for the possibility of HEPD-02 joining the fleet of instruments used for GRBs observation.

Nonetheless, BGO has been discarded for the Limadou HEPD-02 energy detector, in favor of LYSO scintillating crystals, which have fast decay time $(\simeq 40 \mathrm{~ns})$ and can be efficiently readout with the same electronics used for trigger plastic scintillators. To make HEPD-02 sensitive to GRBs means to devise an effective strategy to detect high-energy photon transients over the high background of LYSO crystals. In fact, LYSO features very-high intrinsic radioactivity, about 39 $\mathrm{kBq} / \mathrm{kg}$, due to the presence of ${ }^{176} \mathrm{Lu}$. This level of radioactivity makes it impossible to trigger on fast transients of low-energy photons, while it does not affect the measurement above few MeV.

\section{LYSO background}

The primary goal of this work is to characterise the radioactive background spectrum of LYSO, in order to evaluate the minimum detectable GRB energy for a given sensitivity. LYSO is a Lutetium based, Cerium doped, scintillating crystal with elemental composition $\mathrm{Lu}_{1.8} \mathrm{Y}_{.2} \mathrm{SiO}_{5}$ : $\mathrm{Ce}$. It provides excellent scintillating properties: high density $\left(7.1 \mathrm{~g} / \mathrm{cm}^{3}\right)$, fast decay time $(40 \mathrm{~ns})$ and very-high light output $(\sim 27600 \mathrm{ph} / \mathrm{MeV})$. It naturally contains the radioactive Lutetium isotope ${ }^{176} \mathrm{Lu}\left(2.6 \%\right.$ ab., $\left.T_{1 / 2}=3.78 \times 10^{10} \mathrm{y}\right)$, which decays $\beta^{-}$giving: ${ }^{176} \mathrm{Lu} \longrightarrow{ }^{176} \mathrm{Hf}^{*}+e^{-}+\bar{v}_{e}[5]$. The produced ${ }^{176} \mathrm{Hf}$ is in an excited state that de-excites by emitting three $\mathrm{X}$-rays at $306.8 \mathrm{keV}, 201.8$ $\mathrm{keV}, 88.3 \mathrm{keV}$ (prob. $99.61 \%$ ) or by emitting also a fourth ray at $401 \mathrm{keV}$ (prob. $0.39 \%$ ). The electron can be easily contained in the crystal, whereas each photon can interact via photoelectric or Compton effect, for an overall spectrum like the one in figure 2.

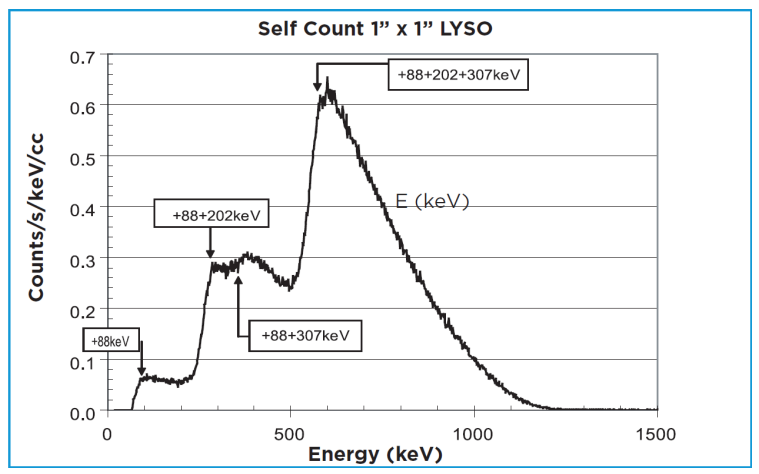

Figure 2: LYSO radioactive background spectrum for a small crystal [6]

It is possible to identify four peaks/shoulders: the largest one at about $600 \mathrm{keV}$, corresponding to events where all the three gamma rays are totally contained in the crystal, the second and the third ones corresponding respectively to the escape of $202 \mathrm{keV}$ and $307 \mathrm{keV} \gamma \mathrm{s}$ from the crystal, and the fourth one corresponding to the escape of both of them. 


\section{Experimental set-up}

To validate the Monte Carlo simulation tool developed to estimate the sensitivity of HEPD-02 to GRBs, the ${ }^{176} \mathrm{Lu}$ background spectrum was measured for a HEPD-01 spare crystal. The set up was composed by a LYSO crystal $\left(4 \times 5 \times 5 \mathrm{~cm}^{3}\right)$, wrapped in mylar foil to maximize the light collection, readout by two PMTs. Each PMT was coupled to a $50 \times 50 \mathrm{~mm}^{2}$ LYSO face and the first PMT (hereafter PMT1) was an Hamamatsu R5946 (diameter 38 mm, operated at -2000 V) while the second (hereafter PMT2) was an Hamamatsu R9880-210 (diameter $16 \mathrm{~mm}$, operated at -850 V). PMT2 was a spare of HEPD-02 and it was operated at operating voltage of HEPD-02. Before closing the box containing the set up, a measurement of the central LYSO face was taken to align the sources used for calibration purposes.

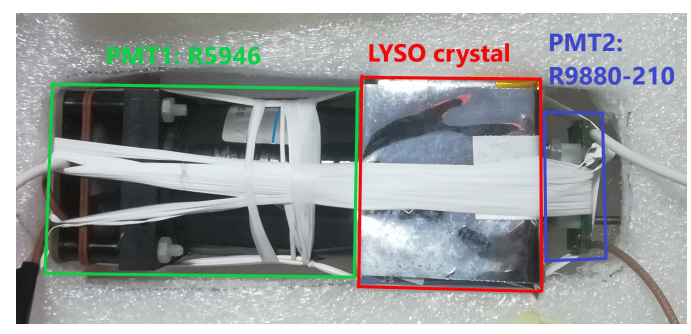

Figure 3: LYSO set up for calibration purposes

The PMT signals were readout by an oscilloscope HDO9104-MS, and the set-up was optimized to measure the low-energy LYSO spectrum by using a PMT with higher energy resolution and by requesting the time coincidence to suppress accidental noise. For each pulse, the maximum peak amplitude, the pulse area and the time difference at half-height was acquired. Table 1 reports the sources used for calibration purposes and the event number collected for each run.

\begin{tabular}{cc} 
Source & Collected events \\
\hline LYSO intrinsic & 123015 \\
${ }^{241} \mathrm{Am}$ & 16929 \\
${ }^{137} \mathrm{Cs}$ & 35270 \\
${ }^{241} \mathrm{Am}+{ }^{137} \mathrm{Cs}$ & 34350 \\
\hline
\end{tabular}

Table 1: Source type and collected events.

\section{Energy calibration}

The first step in data analysis was to define some quality cuts exploiting the relations between the peak area and maximum amplitude, between the time difference and the peak amplitude and between the areas of the waveform of each PMT. In this way, the LYSO raw spectrum was obtained. From raw data, no signal from the sources $\left(333 \mathrm{kBq}{ }^{137} \mathrm{Cs}\right.$ and $\left.74 \mathrm{kBq}{ }^{241} \mathrm{Am}\right)$ was directly observable, because of the high LYSO intrinsic background. Due to lack of more active sources, a simple Geant4 ([7], [8], [9]) simulation was carried out to proceed with the energy calibration.

The simulation considered only the LYSO crystal by setting the geometry of the detector as a box 
with edges $50 \mathrm{~mm}$ and height $40 \mathrm{~mm}$. The sources were simulated by placing them $2 \mathrm{~cm}$ over the LYSO face and neglecting the Lutetium contained in the scintillator. Detector efficiency and energy resolution were added afterwards, whereas the effects of surrounding materials were not included. Subsequently, the energy resolution was added to the spectrum, assuming it dominated by photoelectron Poisson statistics:

$$
\frac{\sigma_{E}}{E}=\frac{k}{\sqrt{E}}
$$

$k^{-2}$ corresponds to the mean number of photoelectrons $/ \mathrm{MeV}$. By fitting the ${ }^{137} \mathrm{Cs}$ peak after subtracting the LYSO background, $k=0.086 \mathrm{MeV}^{1 / 2}$ is obtained, that gives $\sim 130$ p.e. $/ \mathrm{MeV}$ for PMT1. Now it is possible to determine the energy calibration function by relating energy and peak maximum amplitude. This is done by associating spectrum features to the corresponding energy. However, the source spectra were not easily identified due to the LYSO intrinsic radioactivity: by subtracting the LYSO background it is possible to recognize a bump for ${ }^{241} \mathrm{Am}(59.5 \mathrm{keV})$ and a peak for ${ }^{137} \mathrm{Cs}(662 \mathrm{keV})$. Moreover also the $\sim 450 \mathrm{keV}$ Compton edge of ${ }^{137} \mathrm{Cs}$ and the $\sim 300 \mathrm{keV}$ and $\sim 600 \mathrm{keV}$ shoulders of ${ }^{176} \mathrm{Lu}$ can be used for calibration purposes.

In figure 4 the voltage values of these five calibration features are related to the expected energy using the function:

$$
\log _{10}(V)=p_{0}+p_{1} E+p_{2} E^{2}+p_{3} E^{3}
$$

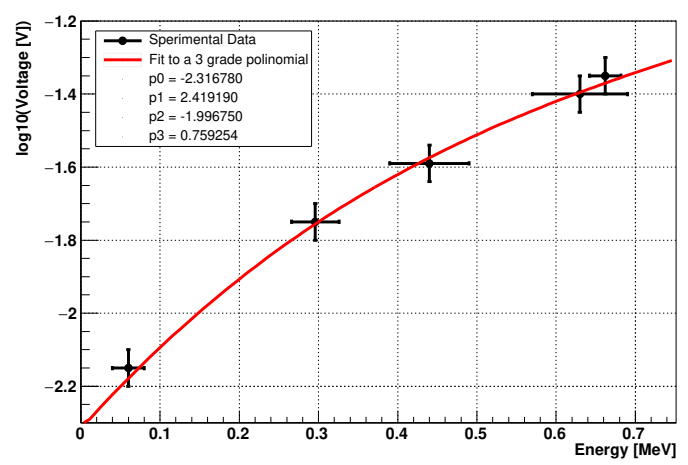

Figure 4: Energy calibration function as compared with some known features in measured energy distributions: (i) ${ }^{241} \mathrm{Am}$ photoelectric peak (59.5 keV); (ii) ${ }^{176} \mathrm{Lu}$ Compton edge (about $~ 300 \mathrm{keV}$ ); (iii) ${ }^{137} \mathrm{Cs}$ Compton edge (about $\sim 450 \mathrm{keV}$ ); (iv) ${ }^{176} \mathrm{Lu}$ Compton edge (about $\sim 600 \mathrm{keV}$ ); (v) ${ }^{137} \mathrm{Cs}$ photoelectric peak $(662 \mathrm{keV})$.

The final determination of function 1 parameters was the result of a global $\chi^{2}$ minimization on the LYSO and ${ }^{241} \mathrm{Am}$ spectrum compared to the Monte Carlo simulations. The fitted spectra for ${ }^{241} \mathrm{Am}$ and LYSO are reported respectively in figure 5 and in figure 6.

The LYSO spectrum is simulated with a reasonable accuracy, although there is a low energy disagreement that was better analysed by calculating the detection efficiency (ratio of measured to expected counts) as a function of the energy. 


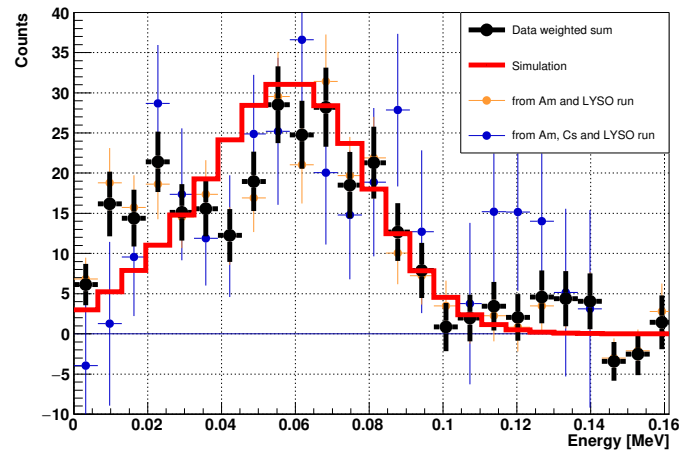

Figure 5: ${ }^{241} \mathrm{Am}$ Spectrum: simulation (red line) and residuals after background subtraction (black points).

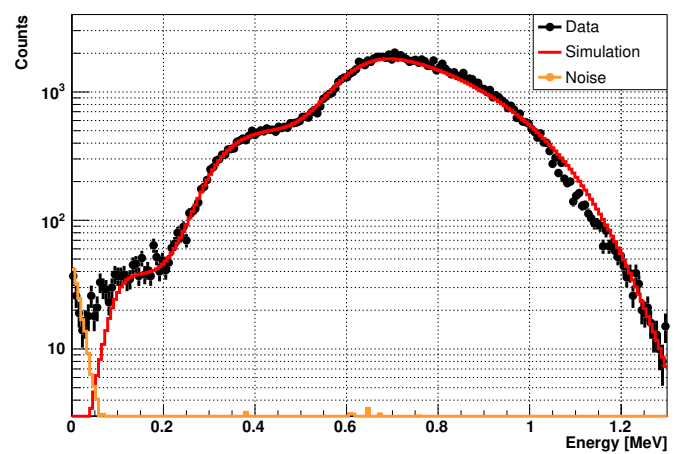

Figure 6: LYSO background spectrum as compared with Geant4 simulation. A residual noise component was identified below $50 \mathrm{keV}$ and fitted with the noise template obtained by rejected data.

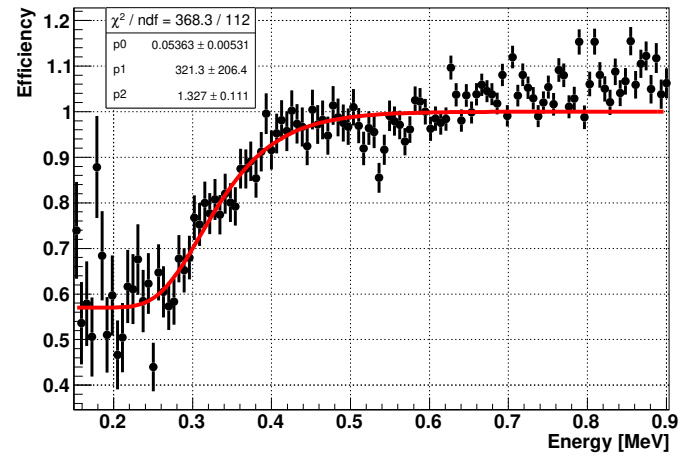

Figure 7: LYSO detector efficiency (described by a sigmoid function)

The main source of this (in-)efficiency at low energy can be identified with the voltage threshold required by the data acquisition system, but other effects can contribute (e.g. a poor description of materials in the Geant4 simulation or a residual electronic noise). 


\section{LYSO expected sensitivity to GRBs}

In the end, it was possible to determine the event rate due to the LYSO background for different energy resolution hypothesis. An energy threshold as large as $1.5 \mathrm{MeV}$ was set, requiring a LYSO background rate less than $1 \mathrm{~Hz}$, and assuming a relative energy resolution of $15 \%$ at $1 \mathrm{MeV}$. Therefore HEPD-02 will be able to detect GRBs with energy larger than $1.5 \mathrm{MeV}$ with a LYSO radioactive background rate of less than $1 \mathrm{~Hz}$. Comparing HEPD-02 with other missions currently taking data, it can be recognised the utility of another detector searching for GRBs in the energy range $2-20 \mathrm{MeV}$, covered only by Fermi, Integral, AGILE and HXMT.

GRBs have been detected in the past in this domain and the HEPD-02 detector will be competitive in this context both to detect long GRB as well as short GRB associated to NS-NS mergers [10].

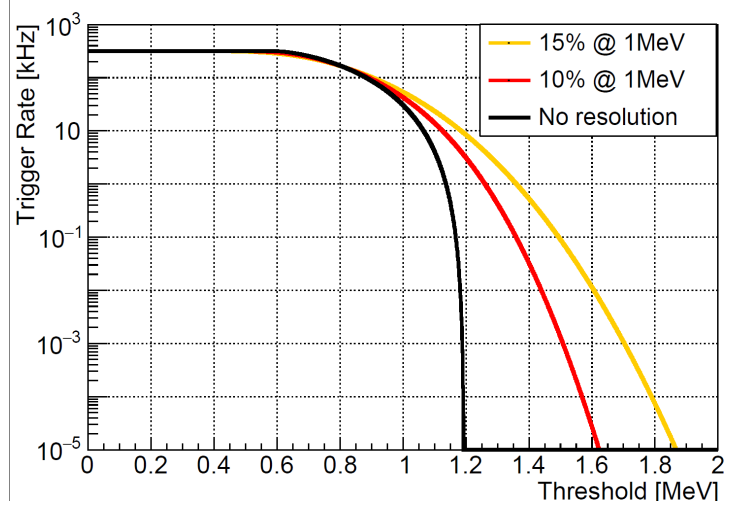

Figure 8: LYSO trigger rate as a function of the energy threshold for different PMTs energy resolution: (i) black line: no energy resolution (ii) red line: $\sigma_{r e l}=10 \%$ at $1 \mathrm{MeV}$ (iii) yellow line: $\sigma_{r e l}=15 \%$ at $1 \mathrm{MeV}$

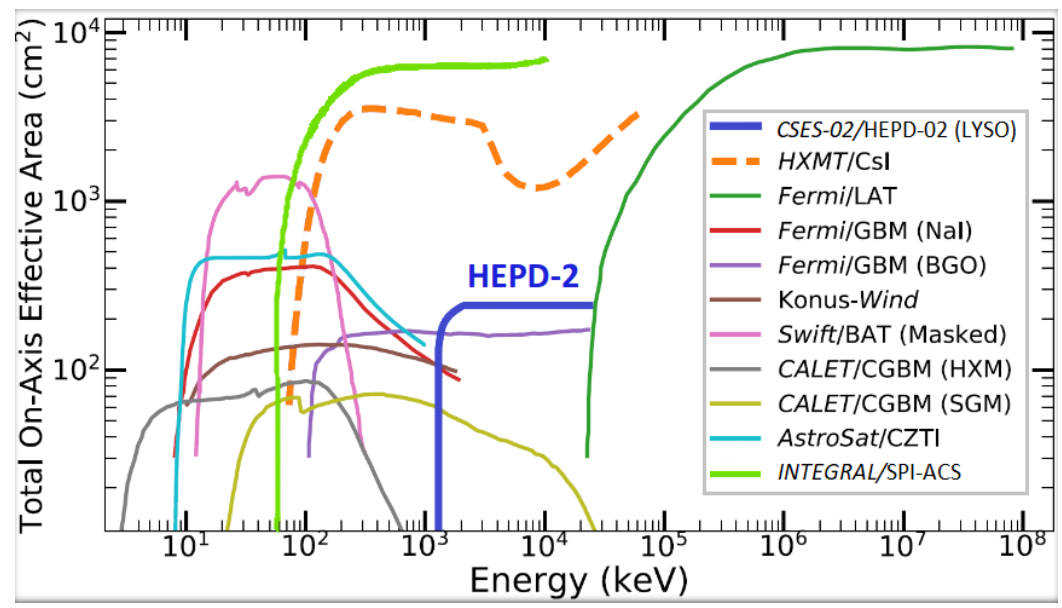

Figure 9: HEPD-02 exposed area to GRBs compared to other missions

\section{Conclusion}

In conclusion, the radioactive background due to ${ }^{176} \mathrm{Lu}$ was measured in a HEPD-01 flight spare scintillation crystal and the energy scale calibration function has been validated from $60 \mathrm{keV}$ 
to $1.3 \mathrm{MeV}$. A preliminary Geant 4 simulation of the detector is able to describe the measured energy spectrum, thus allowing to study the noise effects of ${ }^{176} \mathrm{Lu}$ decay within HEPD-02 detector.

The efficiency function of the detector (related mainly to the used acquisition system) has been measured: we expect a negligible effect for our set-up above $400 \mathrm{keV}$. HEPD-02 will be in condition of measuring photons from GRBs of energy larger than $1.5 \mathrm{MeV}$, with a background rate due to ${ }^{176} \mathrm{Lu}$ of less than $1 \mathrm{~Hz}$. With these features, if equipped with suitable readout electronics, HEPD-02 will be in condition of contributing to the GRB campaign, increasing the sky coverage, also in view of future detection of Gravitational Waves.

\section{References}

[1] Picozza, P. et al., Scientific Goals and In-orbit Performance of the High-energy Particle Detector on Board the CSES, The Astrophysical Journal Supplement Series, 243, 1, 16, 2019 , IOP Publishing.

[2] Abbott, B. et al., Gravitational waves and gamma-rays from a binary neutron star merger: GW170817 and GRB 170817A, The Astrophysical Journal Letters, 848, 2, L13, 2017, IOP Publishing.

[3] Meegan, C. et al., The Fermi gamma-ray burst monitor, The Astrophysical Journal, 702, 1, 791, 2009, IOP Publishing.

[4] Grigoriev, DN et al., Alpha radioactive background in BGO crystals, Nuclear Instruments and Methods in Physics Research Section A: Accelerators, Spectrometers, Detectors and Associated Equipment, 623, 3, 999-1001, 2010, Elsevier.

[5] Brookhaven National Laboratory, National Nuclear Data Center, https://www.nndc.bnl.gov/nudat2/, 2019.

[6] Saint Gobain Crystals, LYSO Scintillation Material, 2004/2017.

[7] Agostinelli, S. et al., GEANT4-a simulation toolkit, Nuclear instruments and methods in physics research section A: Accelerators, Spectrometers, Detectors and Associated Equipment, 506, 3, 250-303, 2003, Elsevier.

[8] Allison, J. et al., Geant4 developments and applications, IEEE Transactions on nuclear science, 53, 1, 270-278, 2006, IEEE.

[9] Allison, J. et al., Recent developments in Geant4, Nuclear Instruments and Methods in Physics Research Section A: Accelerators, Spectrometers, Detectors and Associated Equipment, 835, 186-225, 2016, Elsevier.

[10] Ubertini, P. et al., INTEGRAL search for GW counterparts and the GRB170817A/GW170817 detection, Rendiconti Lincei. Scienze Fisiche e Naturali, 30, 1, 65-70, 2019, Springer. 